\title{
From the new Editor-in-Chief: A sense of personal identity with the Journal of Plant Research
}

\author{
Ikuo Nishida
}

Published online: 4 April 2013

(C) The Botanical Society of Japan and Springer Japan 2013

I would like to introduce myself as the new Editor-in-Chief of the Journal of Plant Research (JPR) for the 4-year term beginning 2 March 2013. I have worked with JPR as an editor in the field of physiology, biochemistry, and molecular biology for the 2009-2012 term under the leadership of Hirokazu Tsukaya, the previous Editor-inChief. As he explained in his editorial in 2013 (Tsukaya 2013), my administrative mission started at this time due to the reform of the Botanical Society of Japan (BSJ) into a Public Interest Corporation. This means that publication of JPR is now recognized as work done by BSJ for the public good, and that BSJ is no longer a society that serves only plant biologists.

JPR has been providing opportunities for publishing across the breadth of plant sciences, from taxonomy, phylogenetics, and evolutionary biology to ecology and ecophysiological and environmental biology; morphology, anatomy, and structural biology; genetics and developmental biology; physiology, biochemistry, and molecular and cell biology; and other related fields. This editorial policy is unique, however, amid the current trend toward publication of special-interest journals, a trend that is beneficial in achieving higher impact factors for those journals and is effective in promoting competitive research. Indeed, each research area mentioned above has a journal of its own, so why do we still need JPR?

In 2011, JPR was recognized as one of the top 100 journals that published influential papers in biology and medicine in the past 100 years (SLA Biomedical \& Life

\section{Nishida $(\square)$}

Division of Life Science, Graduate School of Science and

Engineering, Saitama University, 255 Shimo-Okubo,

Sakura-Ku, Saitama 338-8570, Japan

e-mail: jpreic@gr.saitama-u.ac.jp
Sciences Division 2009). I agree that this recognition is splendid and acknowledges all the efforts of the people concerned with submission, editorial, and publishing (Tsukaya 2009). However, does this alone justify our editorial policy? My opinion is that the philosophy behind our editorial policy is important: no specific area is sufficient to describe the splendid nature of the plant kingdom, and a wider view is always crucial and complementary for better understanding because, as the old saying goes: "You can't see the forest for the trees". This view may be correct ideally, but we do not have much choice when facing a table listing the order of journals' impact factors. I do not mean that JPR will not publish papers that have not qualified for other specific journals. I would just like to remind you that JPR continues to encourage submissions of original research work that can be shared among a readership across the breadth of plant research areas. In this way JPR can provide opportunities for touching on current topics of plant sciences in different fields, which should strengthen a sense of personal identity with JPR as well as with BSJ.

JPR has been supported by hundreds of frequent authors for many years. In this context, it is important for us to encourage young students/postdocs to submit their initial works to JPR. The editors and I declare here our support for young plant scientists through the publication of JPR, keeping in mind the following manuscript preparation guidelines:

1. JPR encourages submissions across the breadth of plant sciences. This means that each author is asked to prepare his/her manuscript so that it will be understandable to a readership outside a specific research area.

2. Those authors whose native languages are not English are requested to submit their manuscripts to 
professional English copy-editing services before submission. I am sure this not only improves the writing skill of authors but also increases the speed of reviewing. In many cases, however, initial editing by senior scientists is important for better manuscript preparation by young authors.

3. Figures and tables are important for the best presentations. In collaboration with Springer, our publisher, JPR is going to provide better tips for figure/table preparation in the near future.

4. We will continue to publish an issue on special topics, with review articles by laureates of BSJ awards including awards for young scientists.

\section{New editors of the 2013-2016 term}

I would also like to introduce the new editors Kouki Hikosaka, Masaki Ito, Maki Katsuhara, Shuichi Sakaguchi and Shinobu Satoh, all of whom have started their missions from the beginning of this year. K. Hikosaka, a professor at Tohoku University, has great expertise in ecology and ecophysiological and environmental biology; M. Ito, an associate professor at Nagoya University, has critical views on molecular and cell biology; M. Katsuhara, a professor at Okayama University, is a specialist in physiology and molecular biology; S. Sakaguchi, an associate professor at Nara Women's University, has morphology, anatomy, and structural biology as his areas of expertise; and S. Satoh, a professor at Tsukuba University, has broad expertise in physiology and biochemistry. In addition to these new members, Akiko Soejima and Toshihiro Yamada continue to be responsible for the research areas of taxonomy, phylogenetics, and evolutionary biology; and Masayoshi Kawaguchi and Hideo Yamazaki are in charge of the research areas in genetics and developmental biology, and physiology and biochemistry, respectively. Submissions to each field are responsibly dealt with by editors who can make final decisions with reference to the exact comments and constructive suggestions of the Editorial Board members or cooperative reviewers. A most conspicuous result was a recent report of our journal's impact factor, which increased from 1.590 (2008) to 1.746 (2011) during the last 4-year term, led by Hirokazu Tsukaya.

To fulfill our missions, it is our great pleasure that Yuko Aoshima, the secretary of JPR, continues to work with me in the Editorial Office of JPR, which recently moved from the University of Tokyo to Saitama University. Together with constant support from all the Editors, Editorial Board members, and Advisory Board members, I am sure that through its publication, JPR will continue to provide the public with the fruits of our labors in plant sciences, which is the mission of BSJ as a Public Interest Corporation and is essential to the sustainability of modern society.

\section{References}

SLA Biomedical \& Life Sciences Division (2009) DBIO: top 100 journals in botany and medicine. http://dbiosla.org/publications/ resources/dbio100.html

Tsukaya H (2009) Journal of Plant Research chosen as one of the top 100 journals on biology and medicine over the past 100 years. J Plant Res 122:353-354

Tsukaya H (2013) On the Journal of Plant Research in the year 2013. J Plant Res 126:1-2 